\title{
Modeling reinforced concrete structures using coupling finite elements for discrete representation of reinforcements
}

\author{
Luís A.G. Bitencourt Jr. ${ }^{a}$, Osvaldo L. Manzoli ${ }^{\text {b,* }}$, Yasmin T. Trindade ${ }^{a}$, \\ Eduardo A. Rodrigues ${ }^{\mathrm{b}}$, Daniel Dias-da-Costa ${ }^{\mathrm{c}}$ \\ ${ }^{a}$ University of São Paulo, Av. Prof. Luciano Gualberto, Trav. 3 n. 380 - CEP 05508-010, São Paulo, SP, Brazil \\ ${ }^{\mathrm{b}}$ São Paulo State University, UNESP/Bauru, Av. Eng. Luiz Edmundo C. Coube 14-01 - CEP 17033-360, Bauru, SP, Brazil \\ ${ }^{\mathrm{c}}$ The University of Sydney, School of Civil Engineering, Faculty of Engineering \& Information Technologies, J05 Civil Engineering Building, 2006 NSW, \\ Australia
}

\section{A R T I C L E I N F O}

\section{Keywords:}

Reinforced concrete

Bond-slip

Coupling finite element

Reinforcing bars

Continuum damage models

\begin{abstract}
A B S T R A C T
This paper presents an alternative methodology to represent rebars and their bond-slip behavior against concrete based on coupling finite elements. Among the main features and advantages of the proposed technique are: (i) the coupling between the two independent meshes for concrete and reinforcement does not introduce any additional degree of freedom in the global problem; (ii) both rigid and non-rigid coupling can be used to represent the particular cases of perfect adherence and general bond-slip behavior, respectively; (iii) rebars of arbitrary geometry and orientation can be modeled; (iv) the methodology can be applied to 2D and 3D problems and (v) the formulation can be adapted to other type of finite elements and implemented easily in any existing FEM code. Constitutive models based on continuum damage mechanics are used to represent the concrete behavior and concrete-rebar interaction. A number of numerical analyses are performed and the results obtained show the versatility and accuracy of the proposed methodology.
\end{abstract}

\section{Introduction}

It is well known that to achieve an accurate and efficient modeling of the nonlinear behavior of reinforced concrete structures by the Finite Element Method (FEM), three important components must be appropriately represented: concrete, steel reinforcement and the bondslip between steel and concrete. Regarding the latter component, since the thickness of the interface between the two materials is usually very small compared to the typical dimensions of the structural member, the computational analysis involves different scales.

A first alternative to model the interface in 3D can be achieved by using the same solid finite elements also employed for the other components - concrete and steel reinforcements. This strategy, however, involves a high computational cost and a complex finite element mesh due to the refinement needed in the neighborhood of the interface. In addition, the cross-section of the steel rebars is much smaller than the general dimensions of the structural member, which precludes using solid elements to represent the reinforcements (see, e.g., Refs. [1,2]) due to the mesh complexity, particularly when the reinforcement ratio of the structural member is considerably large.

To reduce the computational costs of 3D models, a strategy for modeling steel/concrete interaction based on a multi-fiber approach has been proposed by Richard et al. [3]. In their model, the steel/concrete interaction and reinforcement bars are homogenized and the kinematic assumptions are added to relate the global nodal displacement (beam element) to the local strains (cross section). Degenerated finite elements can also be applied to model steel concrete interaction, as employed for Richard et al. [4] for three-dimensional analysis. Recently, interface finite elements with high aspect ratio have been proposed by Rodrigues et al. [5] to describe the interaction between rebars (discretized using one dimensional finite elements) and concrete (represented by threenoded triangular finite elements). In this approach, a continuum damage model is applied to describe the interfaces between the two components. Lastly, one dimensional finite elements are also frequently applied to model both rebars and interfaces. In such models, the behavior of the steel/concrete interface is treated as discrete or embedded formulation to represent the reinforcement bars.

\footnotetext{
* Corresponding author.

E-mail address: omanzoli@feb.unesp.br (O.L. Manzoli).
} 
Smeared, discrete and embedded models can be used to represent the steel rebars. The first model is particularly suitable for modeling distributed reinforcement, since it is based on the assumption that the reinforcements are distributed uniformly with a particular orientation angle over the concrete element. Hence, this formulation is very appealing for modeling concrete structures such as membranes [6,7], shells [8-10] and plates [9-11]. In addition, since it is usually assumed perfect bond between concrete and reinforcements, the constitutive relations are derived using the homogenization theory and, consequently, the reinforcement does not have an explicit discrete representation.

On the discrete and embedded models, the reinforcement layout is explicitly handled. Such models are suitable for applications in which the steel reinforcements are located sparsely. The basic difference among them lies is the way concrete and reinforcement layout are coupled. The discrete model was cited for the first time by Ngo and Scordelis [1]. In this model, the steel reinforcements are connected directly to the adjacent concrete element nodes. Therefore, the major drawback is related to the fact that the concrete mesh does depend on the layout of reinforcements adopted. On the other hand, no special finite elements are required for establishing the connection, since the contribution of the reinforcement to the global stiffness matrix is automatically achieved. E-Mezaini and Çitipitioğlu [12] propose a technique to overcome the problem of mesh dependency, in which isoparametric elements with movable side nodes are applied to concrete, in which case the reinforcement layout can be positioned arbitrarily. This formulation, however, is very limited, since each concrete element cannot be crossed by more than one reinforcement segment. Many researchers employ this formulation when they need to investigate the bond-slip between steel and concrete, since this phenomenon can be introduced straightforwardly. Bond-links [1,2] and contact elements [13] are the most common finite elements used to represent this feature.

In the context of the finite element analysis of reinforced concrete structures, the embedded model seems to be the most appealing, since the discrete rebars can be positioned independently of the concrete mesh. Thus, the rebars can intersect the elements used to represent the concrete in any direction. The contribution of the reinforcement stiffness is superimposed to their parent elements. In the case of perfect bond, the stiffness matrix of the reinforcement (corresponding to its embedded length) is evaluated using the same strain displacement relation of the parent element. It is important to note that, in this case, the size of the stiffness matrix remains unchanged.

In the formulation developed independently by Phillips and Zienkiewicz [14] and Elwi and Murray [15], the embedded reinforcement needs to be aligned with the local isoparametric coordinate axes of the parent elements, bringing some restrictions regarding the reinforcement layout. Chang et al. [16] developed a formulation that allows the reinforcement layer to have an angle relatively to the local isoparametric element axes. However, the reinforcement layer must be straight, and the parent element mesh rectilinear. Elwi and Hrudey [17] published a formulation for curved embedded bars in two-dimensional parent elements that allows slip, whereas Al-Bayati and Fahed [18] developed a procedure to embedded reinforcement in shell elements. Cheng and Fan [19] extended the formulation proposed by Elwi and Hrudey [17] to general three-dimensional elements with improvement and corrections in the transformation process to take into account the reinforcement confinement. Barzegar and Maddipudi [20] developed an automatic procedure for three-dimensional analysis and inclusion of straight segments of embedded reinforcement in a mesh of solid-parametric elements representing concrete. With this procedure only the global coordinates of the nodes need to be provided. Then, an extension of this formulation for modeling of bond-slip in three-dimensional applications is presented by these authors [21]. Markou and Papadrakakis [22] developed an automatic procedure for the generation of embedded steel reinforcement inside hexahedral finite elements to decrease the computational cost in the generation of the input data for the embedded rebar elements.
It is important to consider that in all embedded formulations allowing slip between reinforcement and concrete, the number of degrees of freedom is increased, and consequently, also the size of the stiffness matrix and the computational cost. Another important aspect that should be considered is related to the algorithm necessary to obtain the intersection between the reinforcements and parent elements, and to properly account for the length of reinforcement within each parent element. An integration method or an iterative approach is usually employed to map from global to local the Gauss points coordinates of the reinforcement, and then, evaluate its contribution to the stiffness matrix.

Besides of the approaches mentioned above, nowadays some authors considered the effect of the rebars based on the use of the mixture theory. In this way, Manzoli et al. [23] accounted for the presence of the reinforcing bars oriented in different directions. According to these authors, bundles or layers of rebars, surrounded by concrete, are modeled as composite materials without the need for representing the mesoscopic scale at which the rebars geometrically belong.

In this paper an alternative approach based on the use of coupling finite elements for modeling rebars and their bond-slip relation against concrete are proposed. The model can be classified as a variation of the embedded approach, since both reinforcement layout and concrete are discretized initially in an entirely independent and non-conforming way. Coupling finite elements developed by Bitencourt Jr. et al. [24] are inserted in the mesh to describe the interaction between reinforcements and concrete. This alternative approach is very appealing since it avoids the need for implementing an algorithm to detect the length of the bar embedded in each "parent" element, as is usually the case in existing embedded approaches. Moreover, with the proposed formulation, a concrete element can be crossed by more than one reinforcement segment in any direction automatically. Finally, in order to simulate reinforced concrete structures using the proposed methodology, constitutive models based on the Continuum Damage Mechanics Theory (CDMT) are also formulated for the concrete behavior and rebar interaction.

This paper is organized in five main sections. In section 2 , the strategy used to describe the interaction of the rebars on the concrete matrix based on coupling finite elements is presented. Both cases involving perfect and non-perfect bond conditions are detailed. Then, in section 3 , continuum damage mechanics models are proposed for handling the bond-slip and concrete behavior under tension and compression. Finally, in section 4, several structural examples are presented as means to validate the proposed strategy. The concluding remarks are presented in section 5 .

\section{Discrete representation of rebars}

To present the strategy for the discrete representation of rebars in reinforced concrete structures, let us consider the reinforced concrete corbel in Fig. 1. In such example, the reinforcement layout is quite complex, given that the rebars have different geometries, positioned in distinct directions and completely independent of the concrete mesh. The strategy proposed can be summarized as follows:

1. Concrete mesh discretization based on the geometry of the structural member (Fig. 1(a));

2. Definition of the rebars and corresponding mesh discretization (Fig. 1(b));

3. Definition of coupling finite elements (CFEs) to describe the interaction between concrete and rebars (Fig. 1(c)).

The main novelty of the proposed approach lies in the use of CFEs to couple the two independent and non-conforming meshes for concrete and reinforcement. According to Bitencourt Jr. et al. [24], each CFE has the same nodes of the matching concrete element plus an additional node - herein designated coupling node - represented by the loose node of the rebar that belongs to the domain of the referred concrete element. 


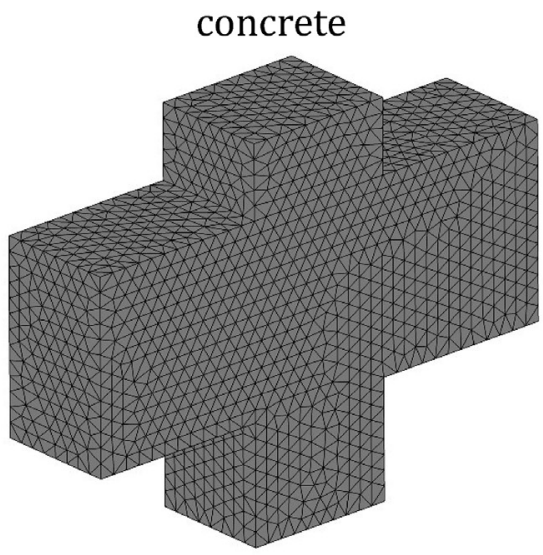

(a)

\section{steel reinforcement bars}

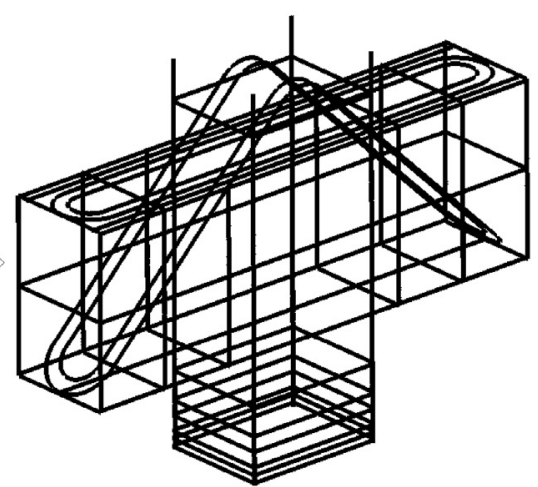

(b)

\section{bars-concrete interaction}

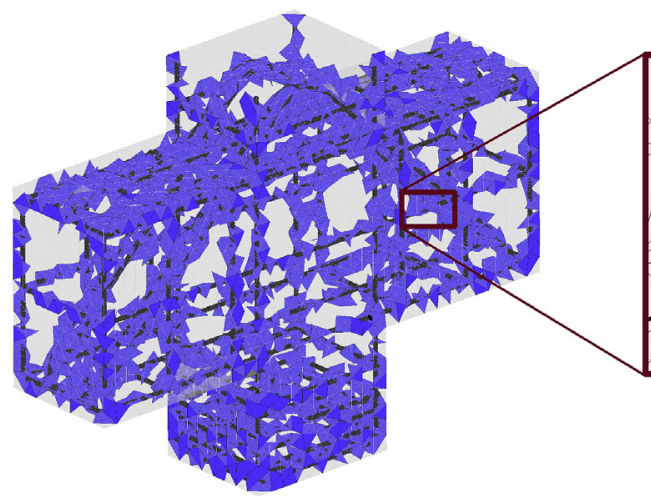

(c)

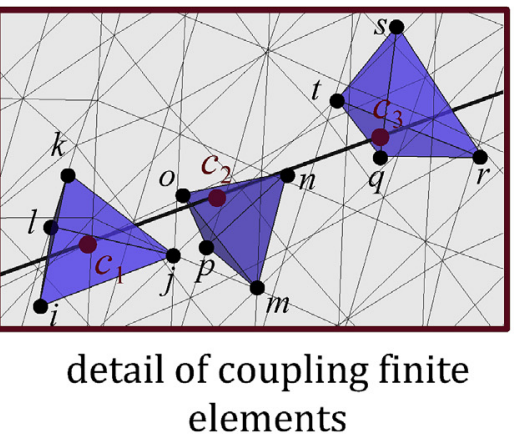

(d)

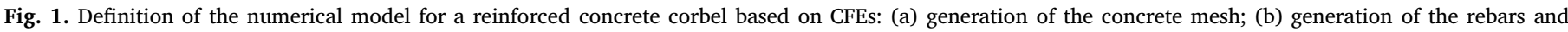
corresponding discretization; (c) coupling procedure; and (d) detail of the CFEs.

As illustrated in Fig. 1(d), for reinforcement nodes $c_{1}, c_{2}$ and $c_{3}$, the following CFEs are created: $C F E_{1}=\left\{i, j, k, l, c_{1}\right\}, C F E_{2}=\left\{m, n, o, p, c_{2}\right\}$, and $C F E_{3}=\left\{q, r, s, t, c_{3}\right\}$. Hence, for each reinforcement node, a CFE is added. It should be highlighted that, as will be discussed later, although new CFEs are introduced in the problem, there is no increase in the degrees of freedom of the global problem.

Since the discretization of concrete and rebars is done entirely independent of each other, the proposed strategy can be classified as a variation of the embedded approaches discussed in the previous section. However, unlike the typical embedded approaches, where the effect of the rebars is locally introduced in the stiffness matrix and internal force vector of the corresponding concrete elements, after the coupling procedure, the contribution of the rebars is added directly to the global internal force vector $\left(\mathbf{F}^{\text {int }}\right)$ and stiffness matrix $(\mathbf{K})$, i.e.:

$$
\mathbf{F}^{\text {int }}=\underbrace{\mathrm{A}_{e=1}^{\text {nel } \Omega^{C}} \mathbf{F}_{e, \Omega^{C}}^{\text {int }}}_{\text {concrete elements }}+\underbrace{\mathrm{A}_{e=1}^{\text {nel } \Omega^{R}} \mathbf{F}_{e, \Omega^{R}}^{\text {int }}}_{\text {rebars }}+\underbrace{\mathrm{A}_{e=1}^{\text {nel } \Omega^{C F E} \mathbf{F}_{e, \Omega^{C F E}}^{\text {int }}}}_{\text {coupling elements }}
$$

and

$$
\mathbf{K}=\underbrace{\mathrm{A}_{e=1}^{\text {nel } \Omega^{C}} \mathbf{K}_{e, \Omega^{C}}}_{\text {concrete elements }}+\underbrace{\mathrm{A}_{e=1}^{\text {nel } \Omega^{R}} \mathbf{K}_{e, \Omega^{R}}}_{\text {rebars }}+\underbrace{\mathrm{A}_{e=1}^{\text {nel } \Omega^{C F E} \mathbf{K}_{e, \Omega^{C F E}}}}_{\text {coupling elements }}
$$

where $A$ stands for the finite element assembly operator. The first and second terms of Equations (1) and (2) are related to the subdomains of concrete, $\Omega^{C}$, and reinforcement, $\Omega^{R}$, respectively, and the third term is associated with the CFEs introduced in the model.

One main advantage of the procedure describe above, is that no algorithm needs to be defined to detect the length of the rebar embed-

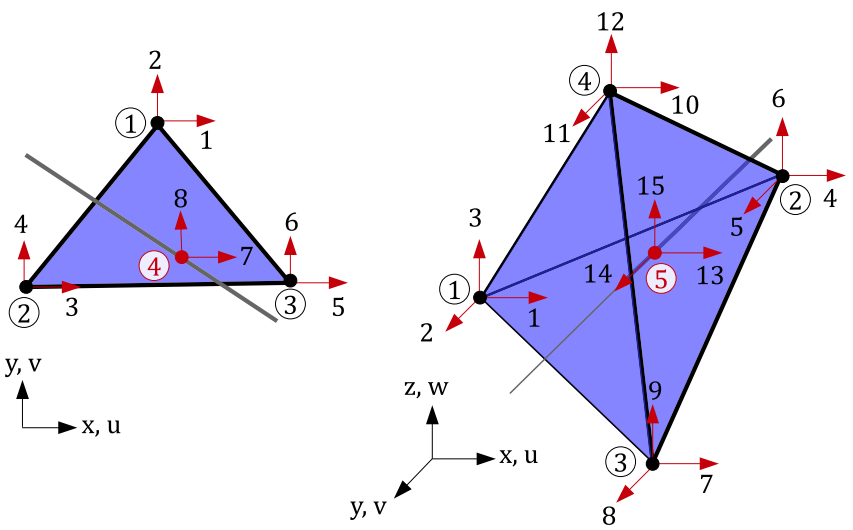

Fig. 2. 2D and 3D CFEs based on linear interpolation functions for displacements: (a) 3-noded triangular element with the $C_{\text {node }}$, and (b) 4-noded tetrahedral element with the $C_{\text {node }}$.

ded in each "parent", as is usually the case for existing embedded approaches. Moreover, with the proposed technique, a concrete element can be automatically crossed by multiple rebar segments in any direction. Finally, the coupling procedure does not introduce any additional degree of freedom in the global problem. In addition, both rigid and non-rigid coupling behaviors can be defined to represent the particular cases of bond behavior, as discussed in the following sections. 


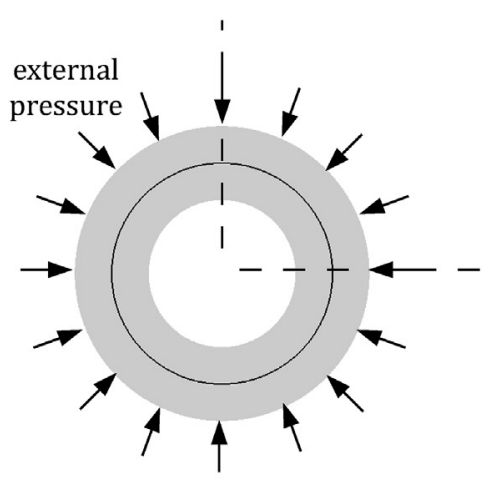

(a)

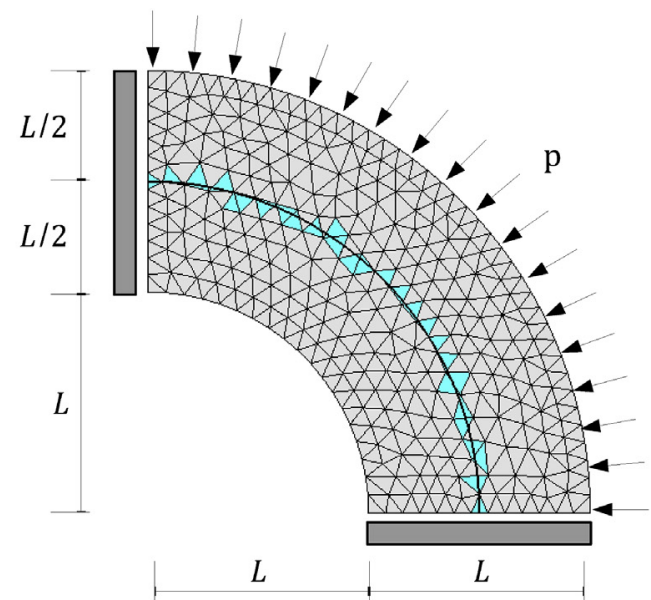

(b)

Fig. 3. Problem with one curved reinforcing layer: (a) global scheme; and (b) numerical model.

\subsection{Concrete-rebar interaction}

The concrete-rebar interaction is described by the use of CFEs. To understand the interaction force introduced by these elements, let us consider a standard isoparametric finite element with domain $\Omega^{e}$, number of nodes equal to $n n$, and shape functions $\bar{N}_{i}(\mathbf{X})(i=1, n n)$, which are defined for the material points $\mathbf{X} \in \Omega^{e}$, such that the displacement, $\overline{\mathbf{U}}$, at any point inside the element domain can be approximated in terms of its nodal displacements, $\mathbf{D}_{i}(i=1, n n)$, as follows:

$\overline{\mathbf{U}}(\mathbf{X})=\sum_{i=1}^{n n} \bar{N}_{i}(\mathbf{X}) \mathbf{D}_{i}$

The CFE is a finite element which has the nodes of the standard isoparametric finite element, plus an additional node $n n+1-$ the coupling node $\left(C_{\text {node }}\right)$ - situated at the material point $\mathbf{X}_{c} \in \Omega^{e}$

Note that, to describe the interaction between the two independent meshes for concrete and reinforcement, the CFEs are constructed using the standard isoparametric elements of the concrete mesh plus the additional node that corresponds to the rebar node located inside their domain. In the implementation presented in this paper, the CFEs for 2D problems all have three nodes from the standard triangular isoparametric element adopted plus the $C_{\text {node }}$; and in 3D, four nodes from the

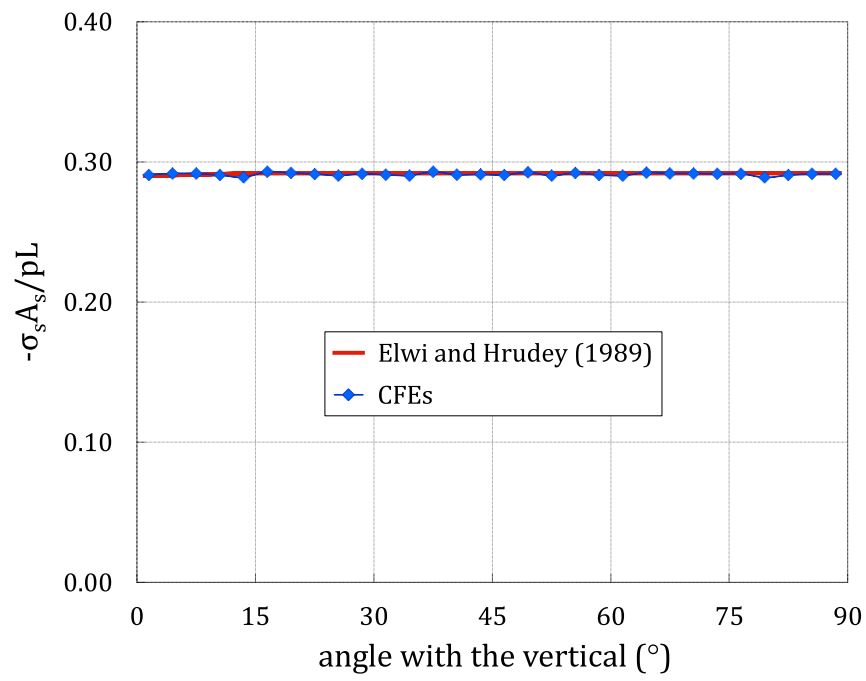

Fig. 4. Steel stress on the model with one curved reinforcing layer. standard tetrahedral isoparametric element adopted plus the $C_{\text {node }}$. This is illustrated in Fig. 2.

The relative displacement, $\llbracket \mathbf{U} \rrbracket$, herein defined by the difference between the displacement of the $C_{\text {node }}$ and the material point $\mathbf{X}_{c}$, can be evaluated using the shape functions of the underlying finite element, $\bar{N}_{i}\left(\mathbf{X}_{c}\right)(i=1, n n)$, as follows:

$\llbracket \mathbf{U} \rrbracket=\mathbf{D}_{n n+1}-\overline{\mathbf{U}}\left(\mathbf{X}_{c}\right)=\mathbf{D}_{n n+1}-\sum_{i=1}^{n n} \bar{N}_{i}\left(\mathbf{X}_{c}\right) \mathbf{D}_{i}=\mathbf{B}_{e} \mathbf{D}_{\mathbf{e}}$,

where matrix $\mathbf{B}_{e}=\left[-\overline{\mathbf{N}}_{1}\left(\mathbf{X}_{c}\right)-\overline{\mathbf{N}}_{2}\left(\mathbf{X}_{c}\right) \ldots-\overline{\mathbf{N}}_{n n}\left(\mathbf{X}_{c}\right) \mathbf{I}\right] ; \overline{\mathbf{N}}_{i}=\bar{N}_{i} \mathbf{I}$; and I is the identity matrix of order 2 or 3 , respectively for $2 \mathrm{D}$ and $3 \mathrm{D}$ problems; and the displacement components of the CFE are stored in $\mathbf{D}_{e}=\left\{\begin{array}{llll}\mathbf{D}_{1} & \mathbf{D}_{2} & \cdots & \mathbf{D}_{n n+1}\end{array}\right\}$.

The internal virtual work of the CFE is given by:

$\delta W_{e}^{\text {int }}=\delta \llbracket \mathbf{U} \rrbracket^{T} \mathbf{F}(\llbracket \mathbf{U} \rrbracket)$,

where $\mathbf{F}(\|\mathbf{U}\|)$ is the reaction force due to the relative displacement $\|\mathbf{U}\|$, and $\delta \llbracket \mathbf{U} \rrbracket$ is an arbitrary virtual relative displacement compatible with the boundary conditions of the problem. Using the same approximation for the virtual relative displacement as the one used for the relative displacement given by Equation (4), i.e., $\delta \llbracket \mathbf{U} \|=\mathbf{B}_{e} \delta \mathbf{D}_{e}$, the internal force vector of the CFE can be expressed as:

$\mathbf{F}_{e}^{\text {int }}=\mathbf{B}_{e}^{T} \mathbf{F}(\llbracket \mathbf{U} \rrbracket)$.

Accordingly, the corresponding tangent stiffness matrix of the CFE is given by:

$\mathbf{K}_{e}=\frac{\partial \mathbf{F}_{e}^{\text {int }}}{\partial \mathbf{D}_{e}}=\mathbf{B}_{e}^{T} \mathbf{C}_{t g} \mathbf{B}_{e}$,

where $\mathbf{C}_{t g}=\partial \mathbf{F}(\llbracket \mathbf{U} \rrbracket) / \partial \llbracket \mathbf{U} \rrbracket$ is the tangent operator of the constitutive relation between reaction force and the relative displacement.

\subsection{Perfect bond}

Perfect bond between concrete and reinforcing steel bars is herein considered by adopting the rigid coupling scheme proposed by Bitencourt Jr. et al. [24]. In this case, the displacement compatibility between the two non-matching meshes is described by a linear elastic relation between the reaction force and the relative displacement see Equation (8). Accordingly, a high elastic stiffness value is assumed for the components $\widetilde{C}$ in the matrix of elastic constants, $\mathbf{C}$ defined in Equation (9):

$\mathbf{F}=\mathbf{C} \llbracket \mathbf{U} \rrbracket=\mathbf{C B}_{\mathbf{e}} \mathbf{D}_{e}$, 


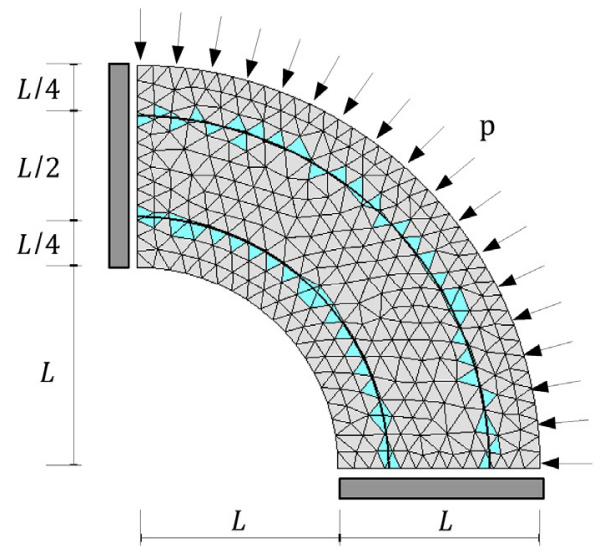

(a)

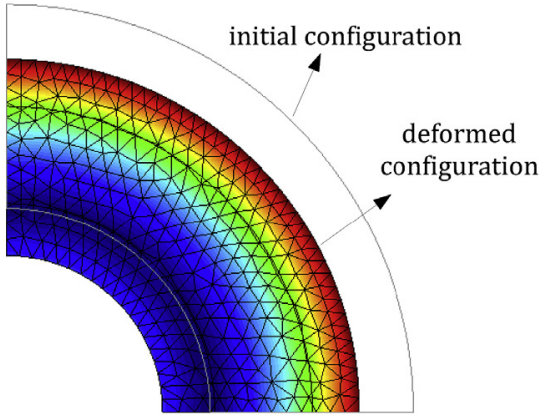

(b)

Fig. 5. Problem with two curved reinforcing layers: (a) numerical model; and (b) total strain distribution.

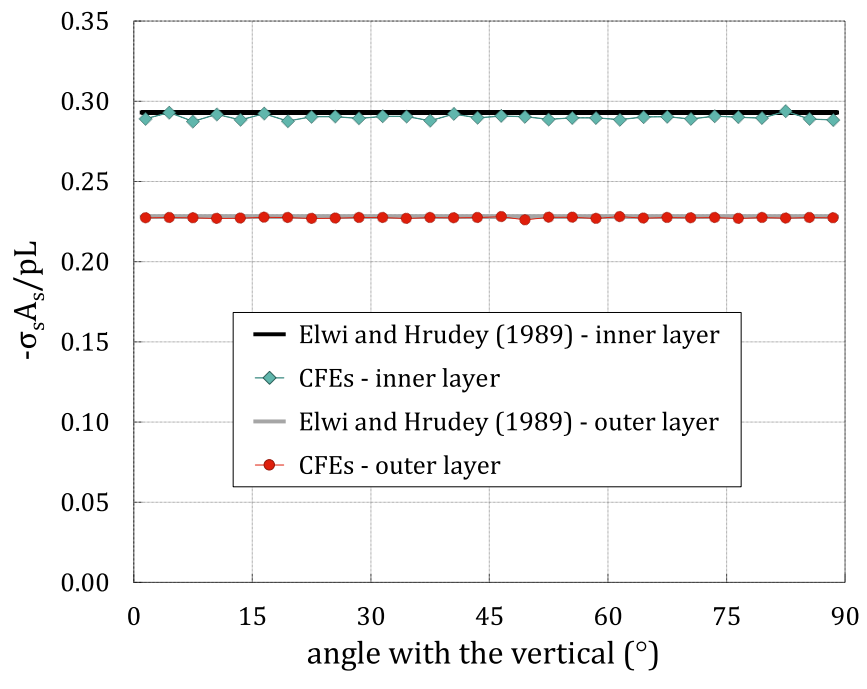

Fig. 6. Steel stress for the model with two curved reinforcing layers.

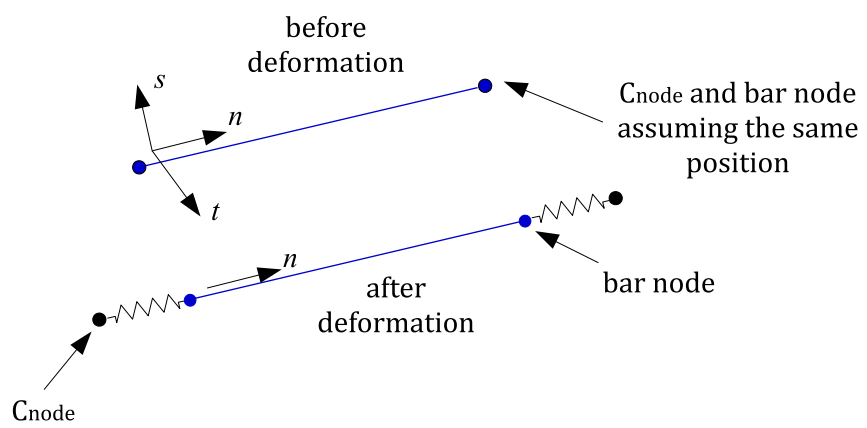

Fig. 7. Reinforcement undergoing slip after deformation.

$\mathbf{C}=\left[\begin{array}{lll}\widetilde{C} & 0 & 0 \\ 0 & \widetilde{C} & 0 \\ 0 & 0 & \widetilde{C}\end{array}\right]$.

It is important to note that $\widetilde{C}$ plays the role of a penalty parameter on the relative displacement, and because of the equilibrium conditions, the interaction force $\mathbf{F}$ in Equation (8) must be bounded. Hence, when the elastic constants tend towards a sufficiently high value, the rela- tive displacement components $\llbracket \mathbf{U} \rrbracket$ approach zero within the normal constraints of the machine precision.

According to Bitencourt et al. [24] the use of $\widetilde{C}$ varying from $\widetilde{C} \cong 10^{6}$ to $\widetilde{C} \cong 10^{9}(\mathrm{~N} / \mathrm{mm})$ is recommended for rigid coupling.

\subsubsection{Example: cylinder with curved reinforcing layers}

A plane strain example is given in this section to illustrate the versatility of the coupling procedure, namely in dealing with curved geometries. The example consists of a thick cylinder subjected to external pressure with curved reinforcing layers, as shown in Fig. 3(a). Based on symmetry conditions, only a quarter of the cylinder is analyzed. The concrete structure is discretized using standard triangular finite elements, whereas the reinforcing layers are independently discretized using two-noded truss elements. The two independent meshes are coupled by four-noded triangular CFEs - see blue elements represented in Fig. 3(b)). The rigid coupling procedure described in subsection 2.2 is adopted using an elastic stiffness of $\widetilde{C} \cong 10^{9} \mathrm{~N} / \mathrm{mm}$. Two cases are considered, with one and two levels of curved reinforcing layers. The materials are considered linear elastic as follows: $A_{s} / L=0.025, E_{s} / E_{c}=8$, and $v=0.25$.

In the following, results are compared with those obtained numerically by Elwi and Hrudey [17] using quarter of thick ring analyses under the same conditions as above.

Fig. 3(b) shows the quarter cylinder model. A total of 562 threenoded triangular finite elements, 30 two-noded truss finite elements, and 31 coupling elements are employed. As can be seen in Fig. 4, the stress distribution along the steel layer is in good agreement with the result by Elwi and Hrudey [17].

The numerical model with two reinforcing layers is depicted in Fig. 5(a). A total of 562 three-noded triangular finite elements, 60 two-noded truss finite elements, and 62 coupling elements are used. In Fig. 5(b), the total strain field is plotted on the deformed configuration, which is in accordance with what is expected for this type of problem.

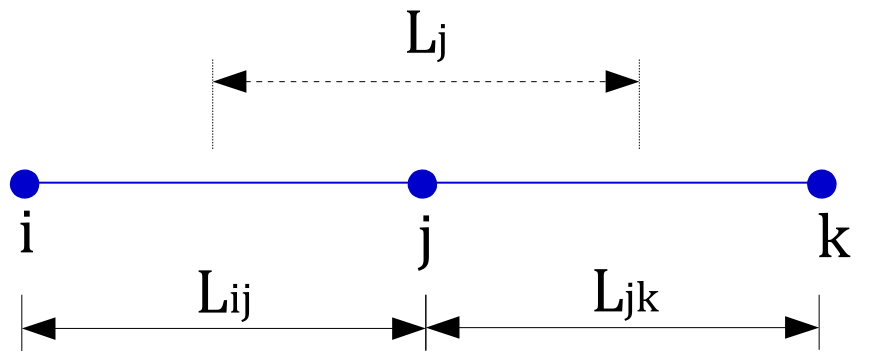

Fig. 8. Influence length of the coupling node. 
Table 1

Ingredients of the continuum damage model for the bond-slip.

\begin{tabular}{ll}
\hline constitutive relation & $\tau_{b}=(1-d) \bar{\tau}_{b}$ \\
effective shear stress & $\bar{\tau}_{b}=c_{n}\left[\left[u_{n}\right]\right]$ \\
damage criterion & $\phi=\left|\bar{\tau}_{b}\right|-r \leq 0$ \\
evolution law of the internal variable & $r=\max \left[\left|\bar{\tau}_{b}\right|\right]$ \\
damage evolution & $d(r)=1-\frac{q(r)}{r}$ \\
\hline
\end{tabular}

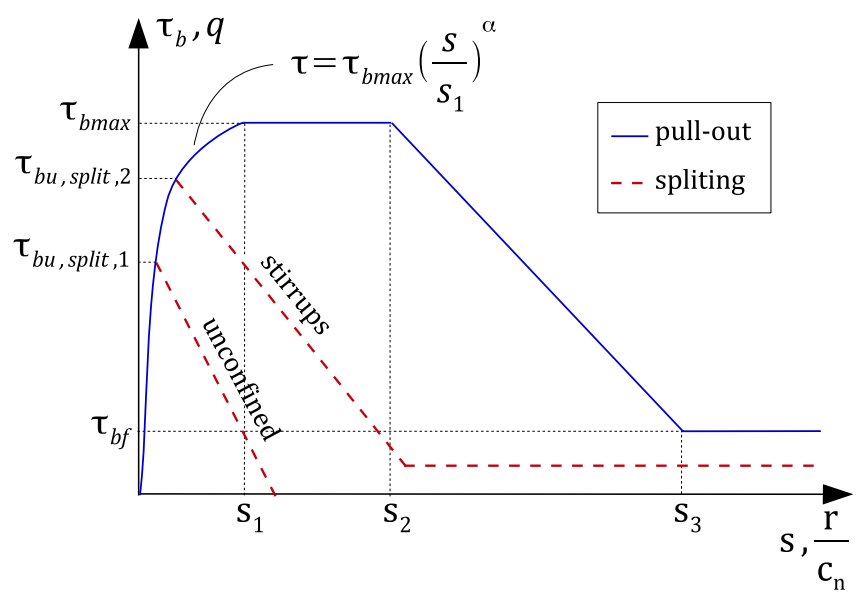

Fig. 9. Interface stress bond-slip relationship (monotonic loading) proposed by the CEB fib Model Code [25].

The graph in Fig. 6 shows the stress distribution along the steel layers (outer and inner). Also for this model, the results are in good agreement with those by Elwi and Hrudey [17].

\subsection{Non-perfect bond}

The non-rigid version of the coupling scheme proposed by Bitencourt Jr. [24]. is used to represent the loss of bond by allowing a relative displacement between concrete and reinforcement. For this type of application, a local system of coordinates, $(\mathbf{n}, \mathbf{s}, \mathbf{t})$, oriented such that axis $\mathbf{n}$ is aligned with the reinforcement must be defined. This later on will allow describing the slip of the reinforcement along its axis against the concrete matrix - see illustration in Fig. 7. Thus, the relative displacement and the corresponding reaction force can be expressed as $[[\mathbf{u}]]=\mathbf{R}[[\mathbf{U}]]$ and $\mathbf{f}=\mathbf{R F}$, respectively, where $\mathbf{R}$ is the orthogonal rotation matrix between local and global reference systems.

The model for the loss of bond can be easily represented by assuming different values for the coupling constants defined in the local coordinate system as follows:

$\mathbf{c}=\left[\begin{array}{ccc}c_{n} & 0 & 0 \\ 0 & c_{s} & 0 \\ 0 & 0 & c_{t}\end{array}\right]$.

To ensure a rigid coupling in the two directions orthogonal to the reinforcement bar (allowing slip only in the direction of the bar), Bitencourt et al. [24] recommend the use of $c_{s}=c_{t}=\widetilde{c}$ varying from $10^{6}$ to $10^{9}(\mathrm{MPa} / \mathrm{mm})$.

In general, bond-slip models establish a relationship between the local (shear) stress, $\tau$, acting at the reinforcement-matrix interface, and the relative displacement (interface slip), $s$. Since the CFE defines an interaction force between the concrete matrix and reinforcement at the coupling node, one may consider that this force results directly from the bond (shear) stress, $\tau_{b}$, on the bonded area (reinforcement-matrix interface) in the neighborhood of the coupling node. Therefore, assum- ing that the bond stress is constant in such neighborhood, and that the influence length contributing to the resultant corresponds to the average of the half distances defined between the node " $j$ " and the adjacent nodes belonging to the reinforcement, " $i$ " and " $k$ " - see Fig. 8 - the interaction force may be expressed as:

$f_{n_{j}}=\tau_{b}\left(\llbracket u_{n_{j}} \rrbracket\right) P L_{j}$,

where $L_{j}=\left(L_{i j}+L_{j k}\right) / 2$ is the influence length and $P$ is the perimeter of the cross-section of the reinforcement. Note that the slip, $s$, is given by the relative displacement along $n$, i.e., $s=\llbracket u_{n} \rrbracket$. Since the shear stress develops in the longitudinal direction of the reinforcement, it only contributes to the interaction force in that same direction. The remaining transverse components of the resultant can be expressed as:

$f_{s_{j}}=\tilde{c} \llbracket u_{s_{j}} \rrbracket P L_{j}$

and

$f_{t_{j}}=\widetilde{c} \llbracket\left[u_{t_{j}} \rrbracket P L_{j}\right.$.

In the following section, a constitutive model based on continuum damage mechanics is used to describe the relationship between the bond stress and relative slip.

\section{Constitutive models}

\subsection{Bond slip}

The main ingredients of the constitutive model herein proposed for the bond-slip behavior are listed in Table 1 , where $c_{n}$ is the elastic stiffness, $d \in[0,1]$ is the scalar damage variable, $\bar{\tau}_{b}$ is the effective shear stress, and $r$ is the strain-like internal variable storing the maximum value ever reached by $\left|\bar{\tau}_{b}\right|$ during the analysis. The function $q(r)$ represents the hardening/softening law of the constitutive model, and it may be adjusted to fit any bond slip model of type $\tau_{b}(s)$ by considering the relationship $q(r)=\tau_{b}\left(r / c_{n}\right)$.

Taking as an example the model proposed by the CEB fib Model Code [25] for monotonic loading (see Fig. 9), the bond stresses, $\tau_{b}$, between concrete and rebar for pullout and splitting failure are given as a function of the relative displacement, $s$, along the axis of the rebar as follows:

$$
\tau_{b}(s)=\left\{\begin{array}{lll}
\tau_{b \max }\left(\frac{s}{s_{1}}\right)^{\alpha} & \text { if } & 0 \leq s \leq s_{1} \\
\tau_{b \max } & \text { if } & s_{1} \leq s \leq s_{2} \\
\tau_{b \max }-\frac{\left(\tau_{b \max }-\tau_{b f}\right)\left(s-s_{2}\right)}{s_{3}-s_{2}} & \text { if } & s_{2} \leq s \leq s_{3} \\
\tau_{b f} & \text { if } & s>s_{3}
\end{array},\right.
$$

and the corresponding hardening/softening law is defined in terms of the stress- and strain-like internal variables as:

$q(r)= \begin{cases}\tau_{b \max }\left(\frac{r / c_{n}}{s_{1}}\right)^{\alpha} & \text { se } 0 \leq r / c_{n} \leq s_{1} \\ \tau_{b \max } & \text { se } s_{1} \leq r / c_{n} \leq s_{2}, \\ \tau_{b \max }-\frac{\left(\tau_{b \max }-\tau_{b f}\right)\left(r / c_{n}-s_{2}\right)}{s_{3}-s_{2}} & \text { se } s_{2} \leq r / c_{n} \leq s_{3} \\ \tau_{b f} & \text { se } r / c_{n}>s_{3}\end{cases}$

where $\alpha, \tau_{b \max }, \tau_{b f}$ and $s_{i}(i=1,2,3)$ are the parameters of the model, and depend on the concrete strength, the geometry of the rebar (ribbed or plain), the confinement provided (confined or unconfined), and on the bond conditions (good, or all other conditions).

\subsubsection{Example: pullout test}

A 3D pullout test is herein presented to assess the behavior of the bar-concrete constitutive interaction defined above. The geometry, boundary conditions and imposed displacement are depicted in Fig. 10. The embedded rebar is discretized using six two-noded truss 


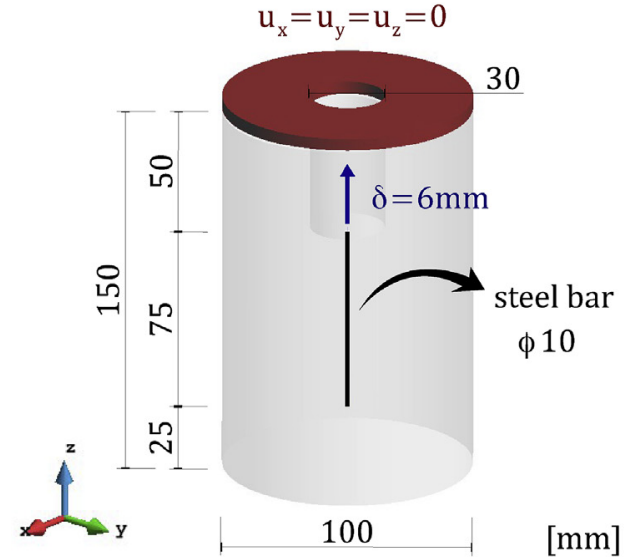

(a)

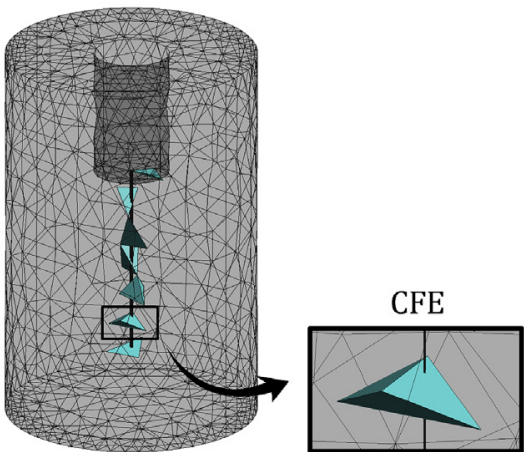

(b) (c)

Fig. 10. Pull-out test set-up (dimensions in $\mathrm{mm}$ ).

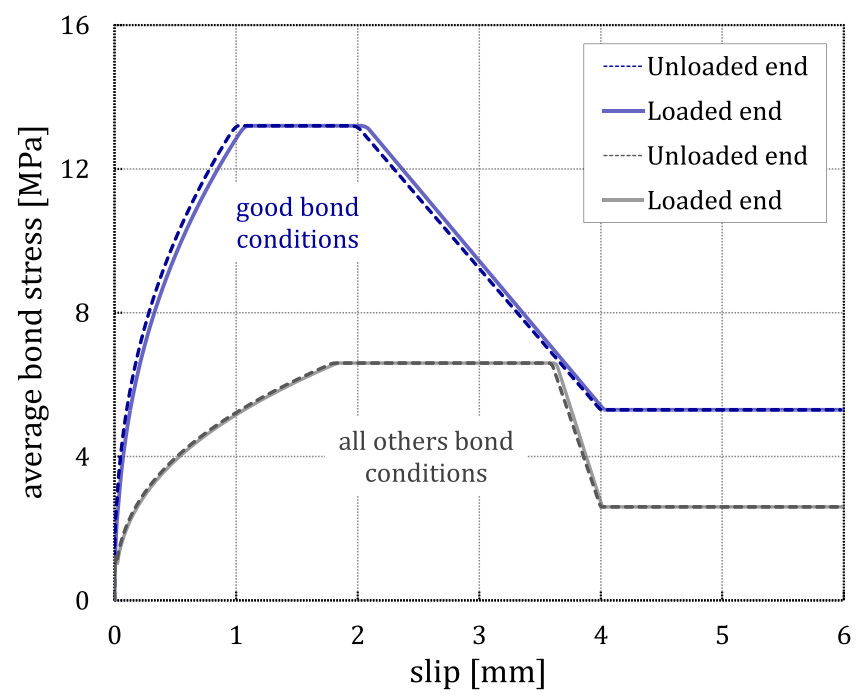

Fig. 11. Evolution of average bond stress with respect to slip at both ends.

elements, whereas for the concrete, 7, 544 four-noded tetrahedral finite elements are used. To couple the two independent meshes, seven fivenoded tetrahedral CFEs are added - see blue elements in Fig. 10.

The concrete cylinder is assumed linearly elastic, with a Young's modulus of $E_{c}=30 \mathrm{GPa}$ and a Poisson's ratio of $v_{c}=0.2$. The steel rebar is elastic perfectly plastic, with a Young's modulus of $E_{s}=200 \mathrm{GPa}$ and a yield stress of $\sigma_{y}=500 \mathrm{MPa}$. The non-rigid coupling procedure is applied to the bond-slip behavior between the materials with a continuum damage model adjusted to the CEB fib Model Code [25].

The constitutive parameters are defined for the two cases by the CEB fib Model Code [25] recommendations for the following pullout failure conditions: good bond conditions $-\tau_{\text {bmax }}=13.2 \mathrm{MPa}, \tau_{b f}=5.3 \mathrm{MPa}$, $\alpha=0.4, s_{1}=1.0 \mathrm{~mm}, s_{2}=2.0 \mathrm{~mm}, s_{3}=4.0 \mathrm{~mm}, c_{n}=10^{3} \mathrm{MPa} / \mathrm{mm}$ and $\tilde{c}=10^{9} \mathrm{MPa} / \mathrm{mm}$; and all other bond conditions $-\tau_{\text {bmax }}=6.6 \mathrm{MPa}$, $\tau_{b f}=2.6 \mathrm{MPa}, \alpha=0.4, s_{1}=1.8 \mathrm{~mm}, s_{2}=3.6 \mathrm{~mm}, s_{3}=4.0 \mathrm{~mm}$, $c_{n}=10^{3} \mathrm{MPa} / \mathrm{mm}$ and $\tilde{c}=10^{9} \mathrm{MPa} / \mathrm{mm}$.

Fig. 11 shows the results obtained for the two cases considered in terms of the average bond stress with respect to the slip at both ends of the bar. As can be seen, the curve proposed by the CEB fib Model Code [25] is properly approximated with only minor differences found between loaded ends.

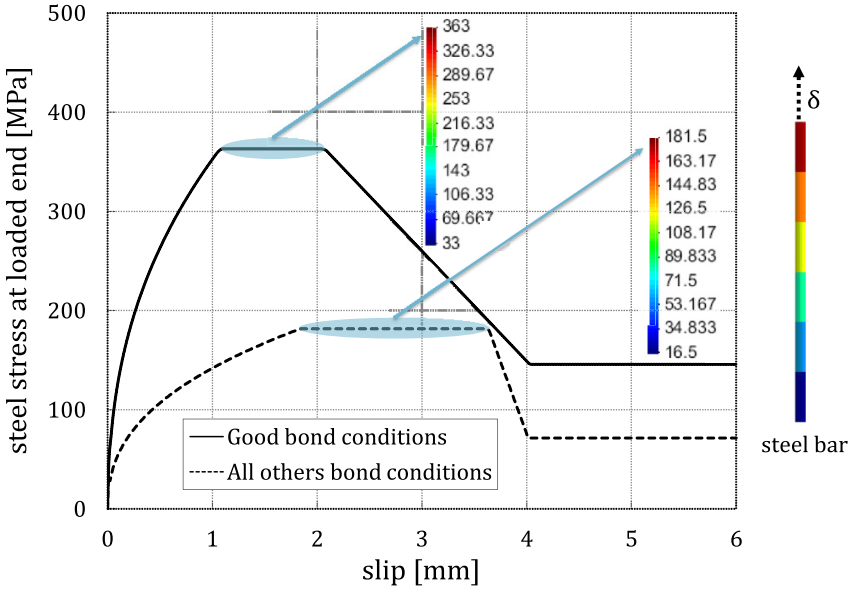

Fig. 12. Steel stress at the loaded end with respect to slip.

The steel stress versus slip at the loaded end is represented in Fig. 12, together with the normal stress distribution along the bar at the maximum shear stress for both cases.

\subsection{Concrete model}

The concrete behavior in the following sections is described by the rate-independent version of the continuum isotropic damage model proposed by Cervera et al. [26]. In this model, the main ingredients are defined separately for tension and compression, respectively using the indices (+) and ( - ). Table 2 summarizes the main features.

In Table 2, $\mathbb{C}$ is the fourth order linear-elastic constitutive tensor; $\sigma$ and $\varepsilon$ are the second order strain and stress tensors, respectively. In addition, after splitting the effective stress tensor in tension and compression components, $\bar{\sigma}_{i}$ denotes the $i$ th principal stress value from tensor $\overline{\boldsymbol{\sigma}}$, and $\boldsymbol{p}_{i}$ represents the unit vector associated with the corresponding principal direction. The symbols $\langle\cdot\rangle$ are the Macaulay brackets. For the equivalent effective compression norm $\overline{\boldsymbol{\tau}}^{-}$, $K=\sqrt{2}(\beta-1) /(2 \beta-1)$ is a material property, which depends on the ratio between the biaxial and uniaxial compressive strengths of the concrete, $\beta$. According to [26], typical values for concrete are $\beta=1.16$ and $K=0.171$. Also in this table, $\bar{\sigma}_{\text {oct }}^{-}$and $\bar{\tau}_{\text {oct }}^{-}$are the octahedral normal and shear stresses, respectively, obtained from $\bar{\sigma}^{-}$. In the definition of the damage criteria, $r^{+}$and $r^{-}$are the strain-like internal variables, which represent the current damage thresholds, being updated continuously to control the size of the expanding damage surface. At the onset 
Table 2

Main features of the continuum isotropic damage model by Cervera et al. [26].

\begin{tabular}{ll} 
constitutive relation & $\boldsymbol{\sigma}=\left(1-d^{+}\right) \overline{\boldsymbol{\sigma}}^{+}+\left(1-d^{-}\right) \overline{\boldsymbol{\sigma}}^{-}$ \\
effective stress tensor & $\bar{\sigma}=\mathbb{C}: \varepsilon$ \\
& $\overline{\boldsymbol{\sigma}}^{+}=\langle\overline{\boldsymbol{\sigma}}\rangle=\sum_{i=1}^{3}\left\langle\overline{\boldsymbol{\sigma}}_{i}\right\rangle \boldsymbol{p}_{i} \otimes \boldsymbol{p}_{i}$ \\
& $\overline{\boldsymbol{\sigma}}^{-}=\bar{\sigma}-\overline{\boldsymbol{\sigma}}^{+}$ \\
\hline equivalent effective norms & $\overline{\boldsymbol{\tau}}^{+}=\sqrt{\overline{\boldsymbol{\sigma}}^{+}: \mathbb{C}^{-1}: \overline{\boldsymbol{\sigma}}^{+}}$ \\
& $\overline{\boldsymbol{\tau}}^{-}=\sqrt{\sqrt{3}\left(K \bar{\sigma}_{\text {oct }}^{-}+\bar{\tau}_{\text {oct }}^{-}\right)}$ \\
damage criterion & $\bar{\phi}^{+/-}\left(\bar{\tau}^{+/-}, r^{+/-}\right)=\bar{\tau}^{+/-}-r^{+/-} \leqslant 0$ \\
evolution law of the internal variable & $r^{+/-}=\max \left(r_{0}^{+/-}, \bar{\tau}^{+/-}\right)$ \\
damage evolution & $d^{+/-}\left(r^{+/-}\right)=1-\frac{q^{+/-}\left(r^{+/-}\right)}{r^{+/-}}$ \\
hardening/softening law & $q^{+}\left(r^{+}\right)=r_{0}^{+} e^{A^{+}\left(1-r^{+} / r_{0}^{+}\right)}$ \\
& $q^{-}\left(r^{-}\right)=r_{0}^{-}\left(1-A^{-}\right)+r^{-} A^{-} e^{B^{-}\left(1-r^{-} / r_{0}^{-}\right)}$ \\
\hline
\end{tabular}
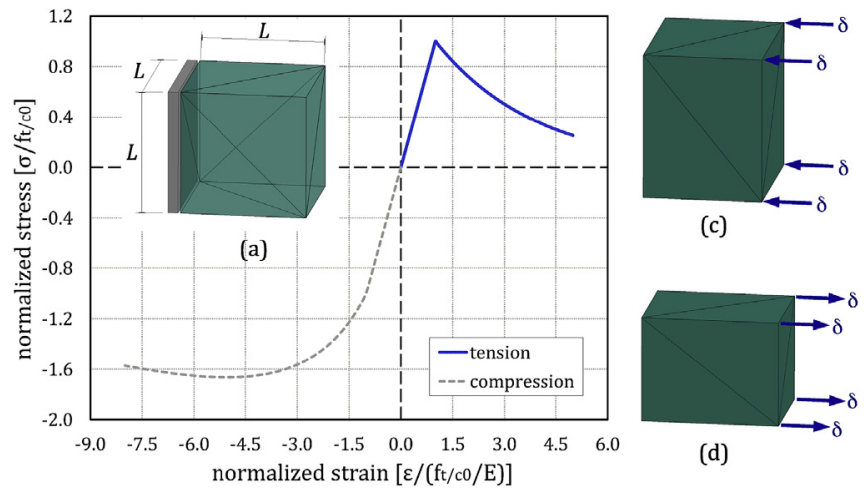

(c)

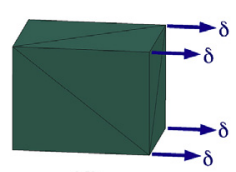

(d)

(b)

Fig. 13. 3D uniaxial tension and compression test: (a) set-up; (b) normalized stress versus strain curve obtained numerically; (c) deformed configuration under compression and (d) under tension loading.

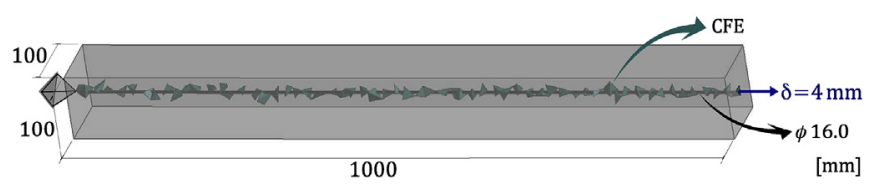

Fig. 14. Tension stiffening example: geometry, boundary conditions, imposed displacement and coupling scheme.

of the analysis, the initial values attributed to the damage thresholds are $r_{0}^{+}=f_{t}$ and $r_{0}^{-}=f_{c 0}$, where $f_{t}$ is the tensile strength and $f_{c 0}$ the compression stress threshold for damage. The two parameters, $A^{-}$and $B^{-}$, are defined so that the stress-strain curve satisfies two previously selected points of the uniaxial compression test.

To satisfy the mesh objectivity condition, the energy dissipated in tension must be properly related to the fracture energy of the material. Therefore, the softening parameter $A^{+}$, is derived from the ratio between the material fracture energy and the characteristic length, $l_{c h}$, such that:

$\frac{1}{A^{+}}=\frac{1}{2 \bar{H}}\left(\frac{1}{l_{c h}}-\bar{H}\right) \geqslant 0$,

where $\bar{H}=f_{t}^{2} / 2 E G_{f}$ is written in terms of the tensile strength $f_{t}$, the elastic modulus $E_{c}$, and the tensile fracture energy $G_{f}$. The characteristic length depends on the spatial discretization and in this paper is assumed to be given by the square root of the finite element area and by the cubic root of the element volume, respectively for 2D and 3D problems.

It should be noted that, from Equation (16), the characteristic length implies a limitation on the maximum size of the finite elements employed in the discretization, since $l_{c h} \leqslant 1 / \bar{H}$.

\subsubsection{Example: uniaxial tension and compression test}

A simple example concerning a 3D uniaxial test is presented in this section to illustrate the constitutive behavior of concrete under tension and compression. Fig. 13(a) shows the test set-up composed by six four-noded tetrahedral finite elements. The parameters adopted are: Young's modulus $E_{c}=30 \mathrm{GPa}$; Poisson's ratio $v=0.2$; tensile strength $f_{t}=2.0 \mathrm{MPa}$; compression stress threshold for damage $f_{c 0}=12 \mathrm{MPa}$; fracture energy $G_{f}=0.25 \mathrm{~N} / \mathrm{mm}$; and the compressive parameters $A^{-}=1.0$ and $B^{-}=0.89$.

The numerical response is depicted in Fig. 13 for both compression and tension loading. As can be concluded, the damage model describes the expected behavior.

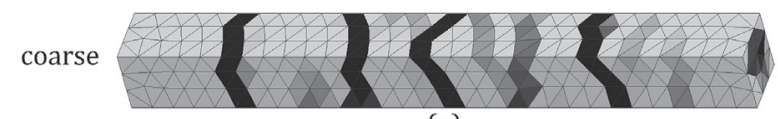

(a)

medium

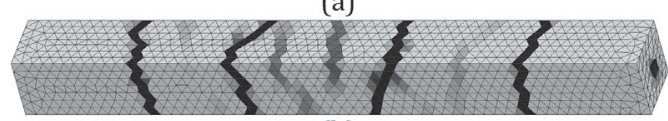

(b)

fine

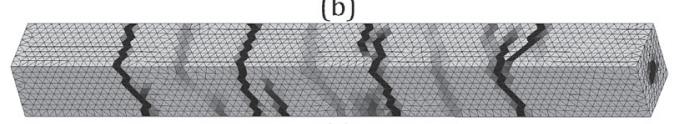

(c)

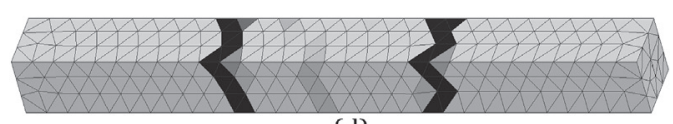

(d)

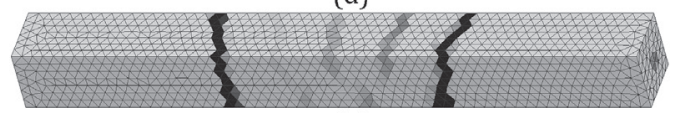

(e)

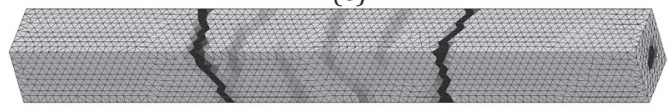

(f)

Fig. 15. Crack pattern for $\delta=2 \mathrm{~mm}$ : good bond conditions (a) coarse mesh, (b) medium mesh, and (c) fine mesh; and all other bond conditions (d) coarse mesh, (e) medium mesh, and (f) fine mesh. 
Table 3

Definition of the three finite element meshes.

\begin{tabular}{lllll}
\hline \multirow{2}{*}{ Type of mesh } & \multicolumn{2}{l}{ Number of elements } & & \\
\cline { 2 - 5 } & two-noded trusses & four-noded tetrahedrals & five-noded tetrahedrals CFEs & total number of elements \\
\hline coarse & 30 & 2617 & 31 & 2678 \\
medium & 60 & 21,528 & 61 & 21,649 \\
fine & 80 & 50,650 & 81 & 50,811 \\
\hline
\end{tabular}

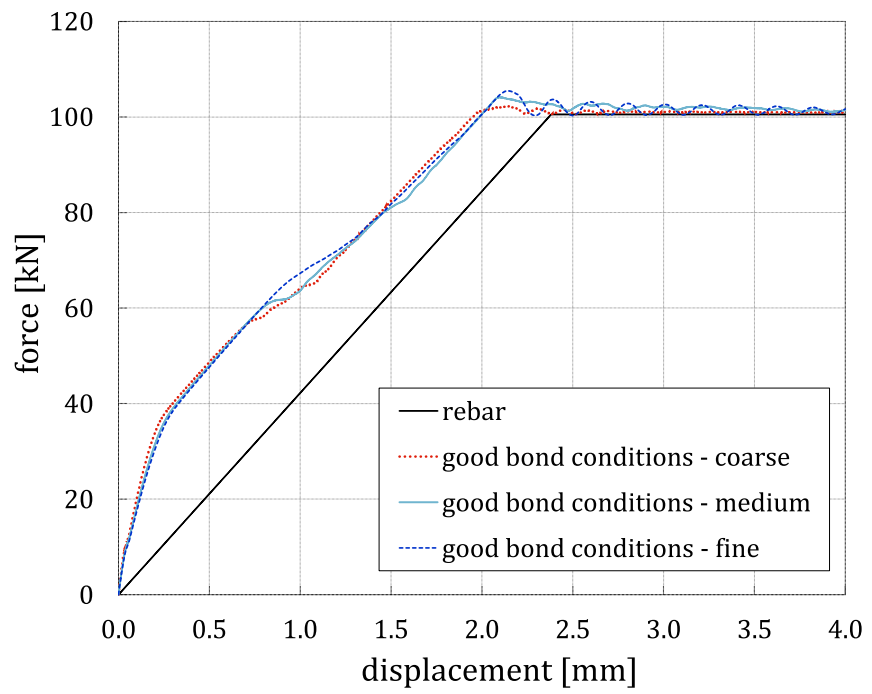

Fig. 16. Tension stiffening test: reaction force versus imposed displacement curves for good bond conditions.

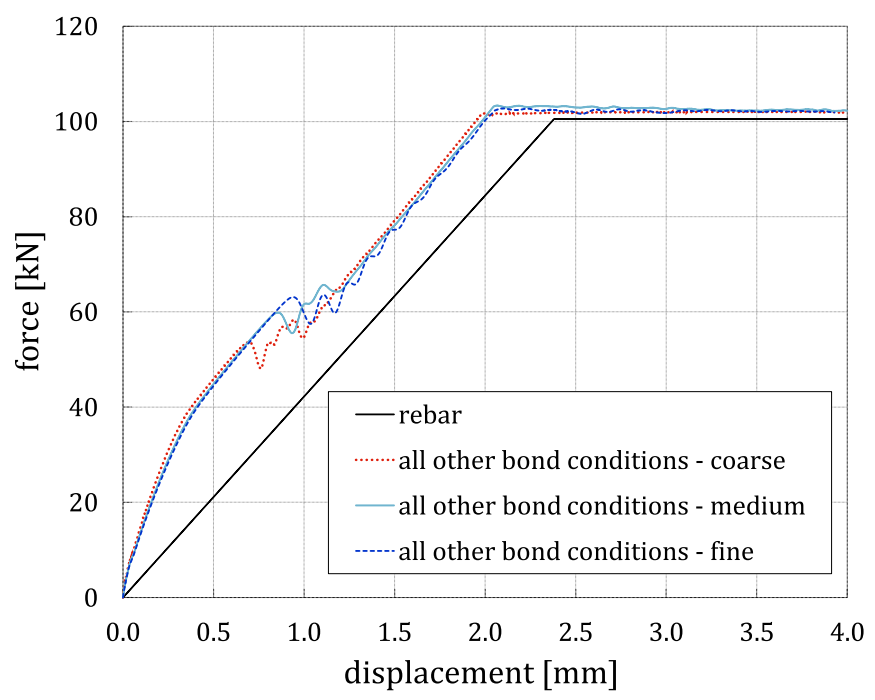

Fig. 17. Tension stiffening test: reaction force versus imposed displacement curves for all other bond conditions.

\section{Numerical applications}

This section presents three complex structural examples selected to demonstrate the advantages of the new coupling technique.

\subsection{Tension stiffening}

In this first example, a tension stiffening test is performed numerically in a reinforced concrete specimen. Focus is given to the mesh

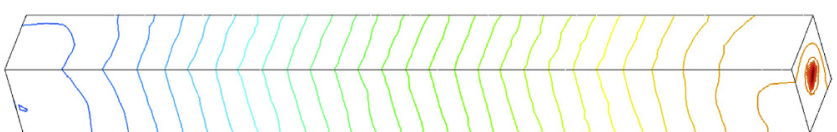

(a)

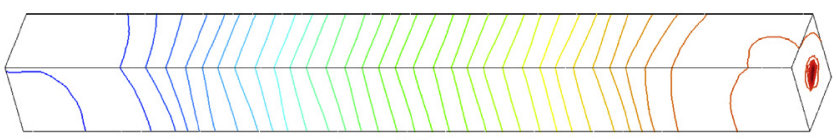

(b)

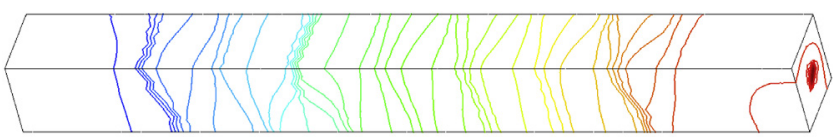

(c)

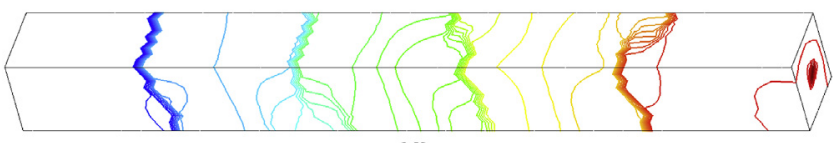

(d)

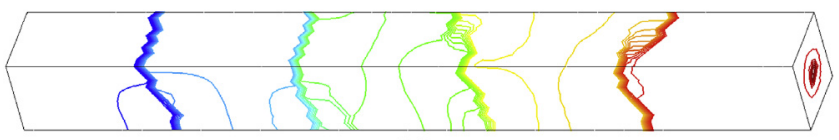

(e)

Fig. 18. Total iso-displacement contour representing the evolution of the crack pattern obtained with the fine mesh and good bond conditions: (a) $\delta=0.2 \mathrm{~mm}$; (b) $\delta=0.6 \mathrm{~mm}$; (c) $\delta=1.0 \mathrm{~mm}$; (d) $\delta=1.4 \mathrm{~mm}$; and (e) $\delta=2.0 \mathrm{~mm}$.

sensitivity of the bond slip model and, consequently, also of the crack spacing.

The geometry, boundary conditions and imposed displacement are illustrated in Fig. 14. The test set-up consists of a concrete prism with a cross-section of $100 \times 100 \mathrm{~mm}^{2}$ and $1000 \mathrm{~mm}$ length. A single rebar with diameter of $\phi=16 \mathrm{~mm}$ is placed along the centroid of the crosssection.

The mesh sensitivity is assessed by considering different discretizations for the rebar and concrete (see Fig. 15). Table 3 summarizes the type and number of elements for the three selected cases (coarse, medium and fine meshes). In all simulations, the concrete behavior is described by the continuum damage model proposed by Cervera et al. [26] with the following parameters: Young's modulus $E=35 \mathrm{GPa}$; Poisson's ratio $v=0.2$; tensile strength $f_{t}=3.0 \mathrm{MPa}$; compression stress threshold for damage $f_{c 0}=19.2 \mathrm{MPa}$; fracture energy $G_{f}=0.25 \mathrm{~N} / \mathrm{mm}$; and the compressive parameters $A^{-}=1.0$ and $B^{-}=0.89$.

The bond-slip behavior between steel and concrete is represented by the non-rigid coupling procedure with the continuum damage model adjusted to the CEB fib Model Code [25], as described in subsection 3.1. Two sets of parameters are defined for the ribbed bar, splitting failure and unconfined conditions, by considering a concrete of $f_{c k}=32 \mathrm{MPa}$ : good bond conditions - $\tau_{b u, s p l i t}=8.8 \mathrm{MPa}, \tau_{b f}=0$, $\alpha=0.4, s_{1}=s_{2}=0.23 \mathrm{~mm}, s_{3}=0.28 \mathrm{~mm}$; for all other bond conditions $-\tau_{\text {bu,split }}=6.16 \mathrm{MPa}, \tau_{b f}=0, \alpha=0.4, s_{1}=s_{2}=0.97 \mathrm{~mm}$, $s_{3}=1.16 \mathrm{~mm}$. In both cases, the following coupling parameters are 


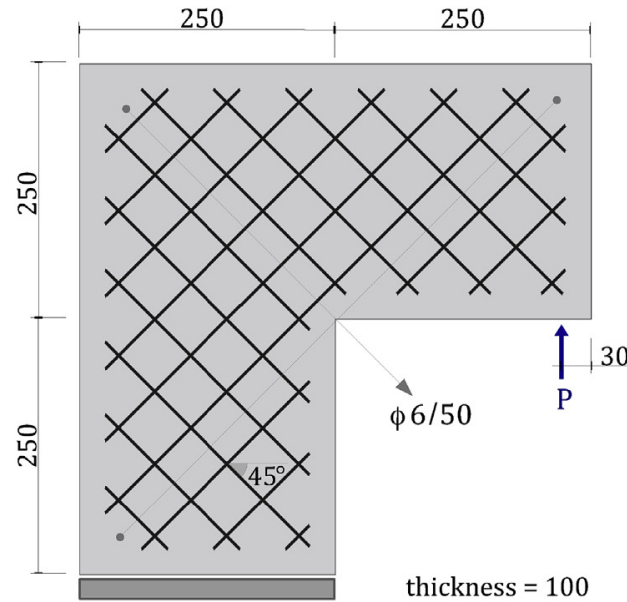

(a)

$[\mathrm{mm}]$

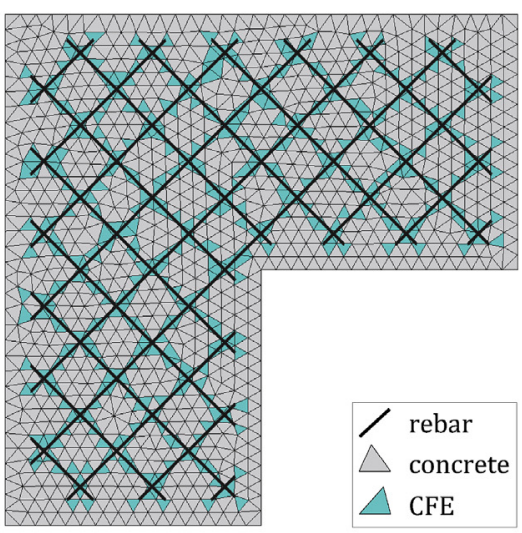

(b)

Fig. 19. L-shaped panel test: (a) set-up; (b) coupling scheme between rebars and concrete.

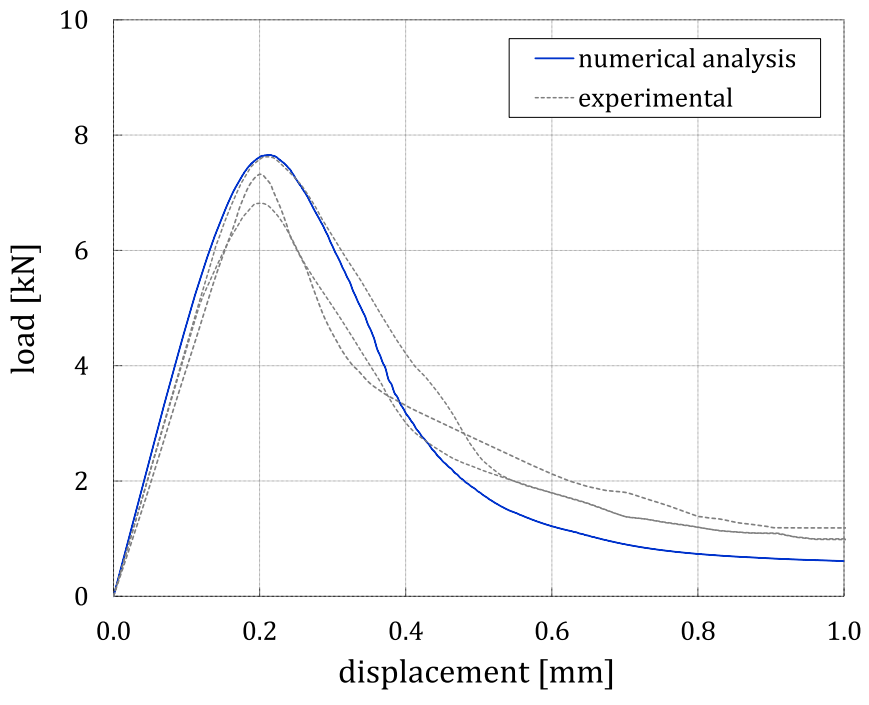

Fig. 20. Plain concrete L-shaped panel: load versus vertical displacement of the loaded point.

adopted: $c_{n}=10^{3} \mathrm{MPa} / \mathrm{mm}$ and $\widetilde{c}=10^{9} \mathrm{MPa} / \mathrm{mm}$.

Fig. 15 shows the crack pattern at $\delta=2 \mathrm{~mm}$ obtained with the three meshes, for both good bond conditions (Fig. 15(a), (b) and (c)) and all other bond conditions (Fig. 15(d), (e) and (f)). It can be concluded that for the employed meshes the results are mesh-independent, since the location and number of cracks remain the same. In addition, as it was already expected from the literature, the number of cracks for good bond conditions is higher.

The reaction force versus imposed displacement is shown in Figs. 16 and 17 , respectively. In all cases, the results are very similar independently of the mesh size.

Finally, the cracking process is plotted in Fig. 18 for different stages of loading using total iso-displacement contours for the model with good bond conditions and fine mesh.

\subsection{L-shaped panels}

The L-shape panels experimentally tested by Winkler et al. [27] are herein numerically simulated using plane stress conditions with an out-

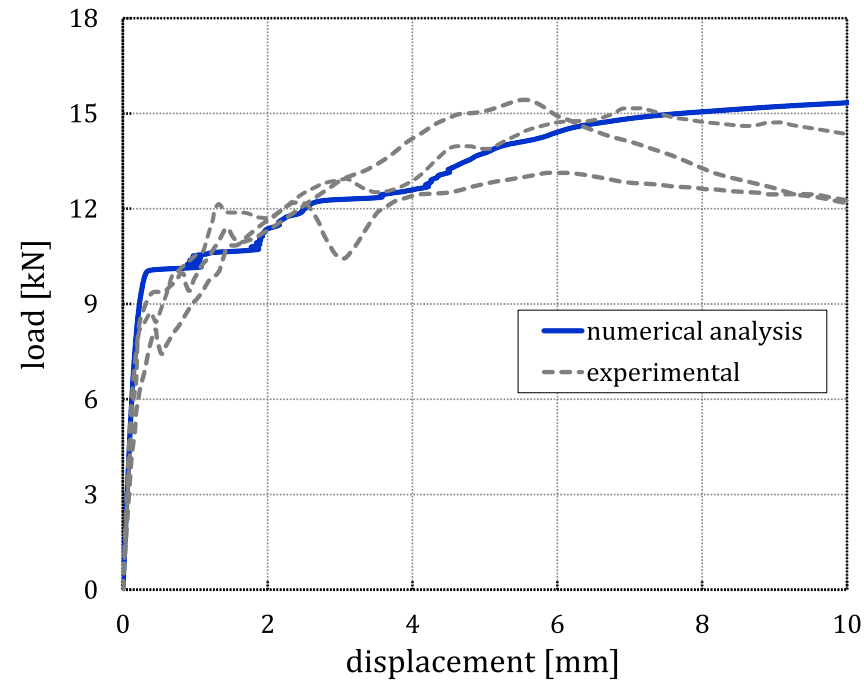

Fig. 21. Reinforced L-shaped panel test: load versus vertical displacement of the loaded point.

of-plane thickness of $100 \mathrm{~mm}$. In this example, two cases are considered: plain concrete and reinforced concrete with a welded orthogonal reinforcing mesh defining an angle of $45^{\circ}$ with the edges of the panel, as illustrated in Fig. 19. The lower horizontal edge is fixed and a vertical load is applied at a distance of $30 \mathrm{~mm}$ from the vertical right edge.

In both panels the concrete is discretized using 1886 three-noded triangular finite elements. The damage model described in section 3.2 is adopted with the following parameters: Young's modulus $E_{c}=20 \mathrm{GPa}$; Poisson's ratio $v=0.18$; tensile strength $f_{t}=2.65 \mathrm{MPa}$; compression stress threshold for damage $f_{c 0}=17.7 \mathrm{MPa}$; fracture energy $G_{f}=0.08 \mathrm{~N} / \mathrm{mm}$; and the compressive parameters $A^{-}=1.0$ and $B^{-}=0.89$.

For the reinforced L-shaped panel, the rebars are discretized using 456 two-noded truss finite elements. The rebars are assumed elastic perfectly plastic, with a Young's modulus of $E_{s}=179.1 \mathrm{GPa}$ and a yield stress of $\sigma_{y}=526.3 \mathrm{MPa}$. Perfect bond conditions are assumed using 480 five-noded tetrahedral coupling finite elements (see Fig. 19(b)) with elastic coupling parameters of $\widetilde{C}=10^{9} \mathrm{~N} / \mathrm{mm}$.

The numerical results regarding both applied load versus displacement curve and crack propagation are compared with experimental data. Figs. 20 and 21 show the curves obtained for the plain and rein- 


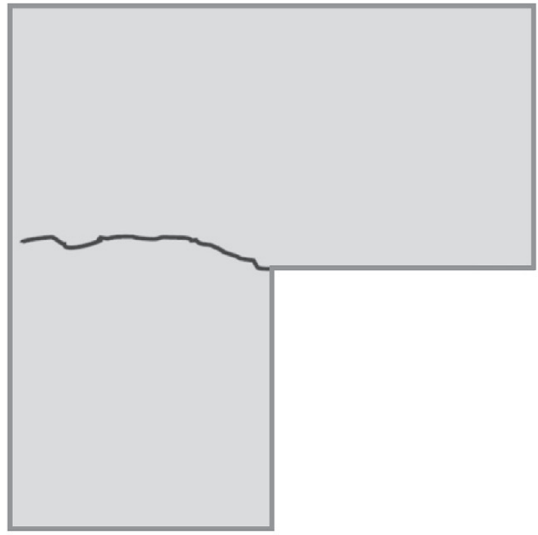

(a)

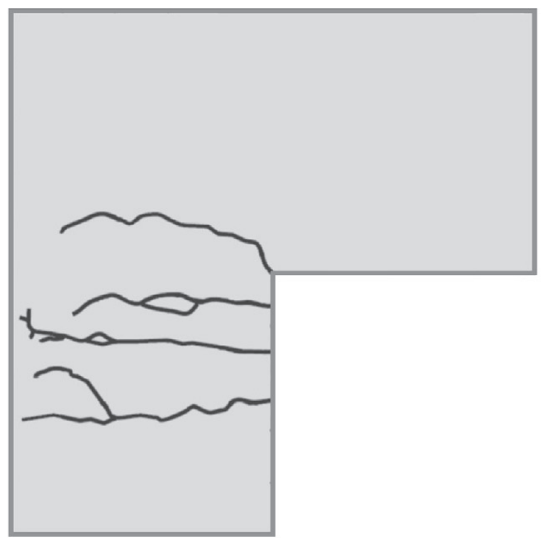

(c)

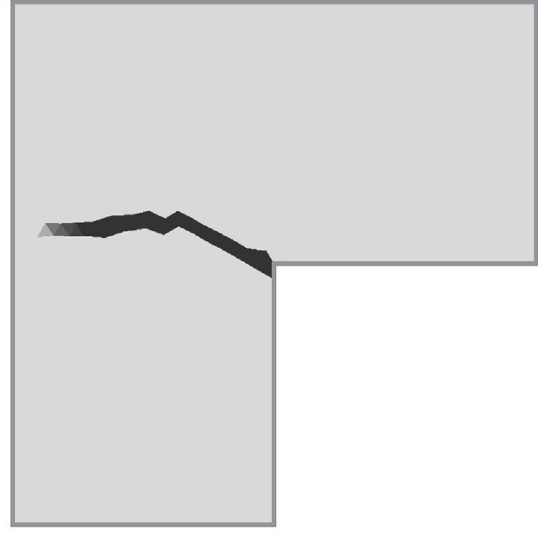

(b)

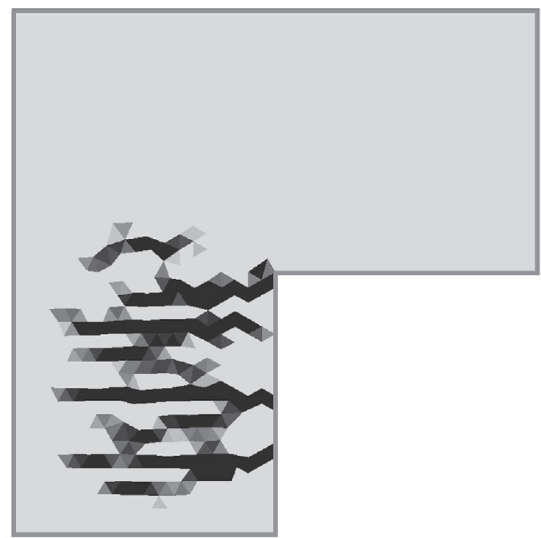

(d)

Fig. 22. Crack propagation: plain concrete (a) experimental and (b) numerical; and for reinforced concrete (c) experimental; and (d) numerical.

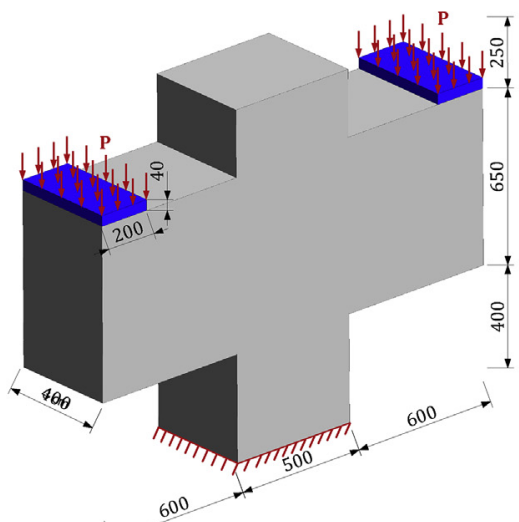

(a)

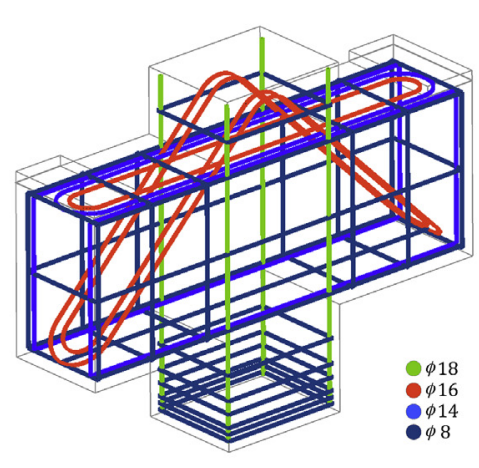

(b)

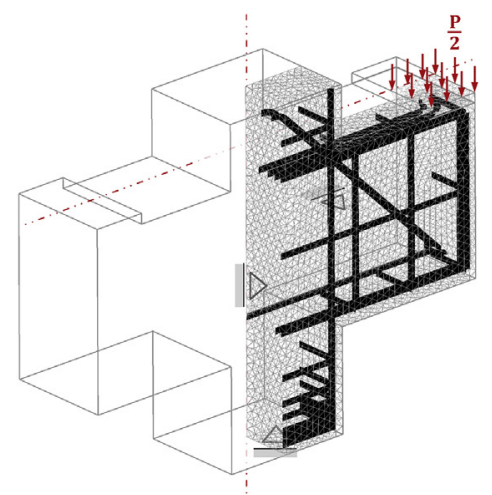

(c)

Fig. 23. Reinforced concrete corbel: (a) set-up; (b) reinforcement layout; and (c) symmetry conditions applied.

Table 4

Material parameters for the concrete damage model.

\begin{tabular}{ll}
\hline Young's modulus & $E=21.87 \mathrm{GPa}$ \\
Poisson's ratio & $v=0.2$ \\
Tensile strength & $f_{t}=2.26 \mathrm{MPa}$ \\
Compression stress threshold for damage & $f_{c_{0}}=18.0 \mathrm{MPa}$ \\
Fracture energy & $G_{f}=0.1 \mathrm{~N} / \mathrm{mm}$ \\
Compressive parameters & $A^{-}=1.0$ and $B^{-}=0.89$ \\
\hline
\end{tabular}

Table 5

Material parameters for the steel reinforcement.

\begin{tabular}{ll}
\hline Young's modulus & $E_{s}=206 \mathrm{GPa}$ \\
Yield stress & $\sigma_{y}=430 \mathrm{MPa}$ \\
\hline
\end{tabular}




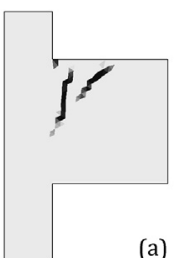

(a)

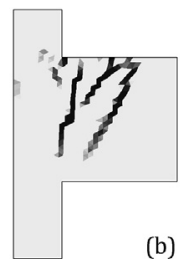

(b)
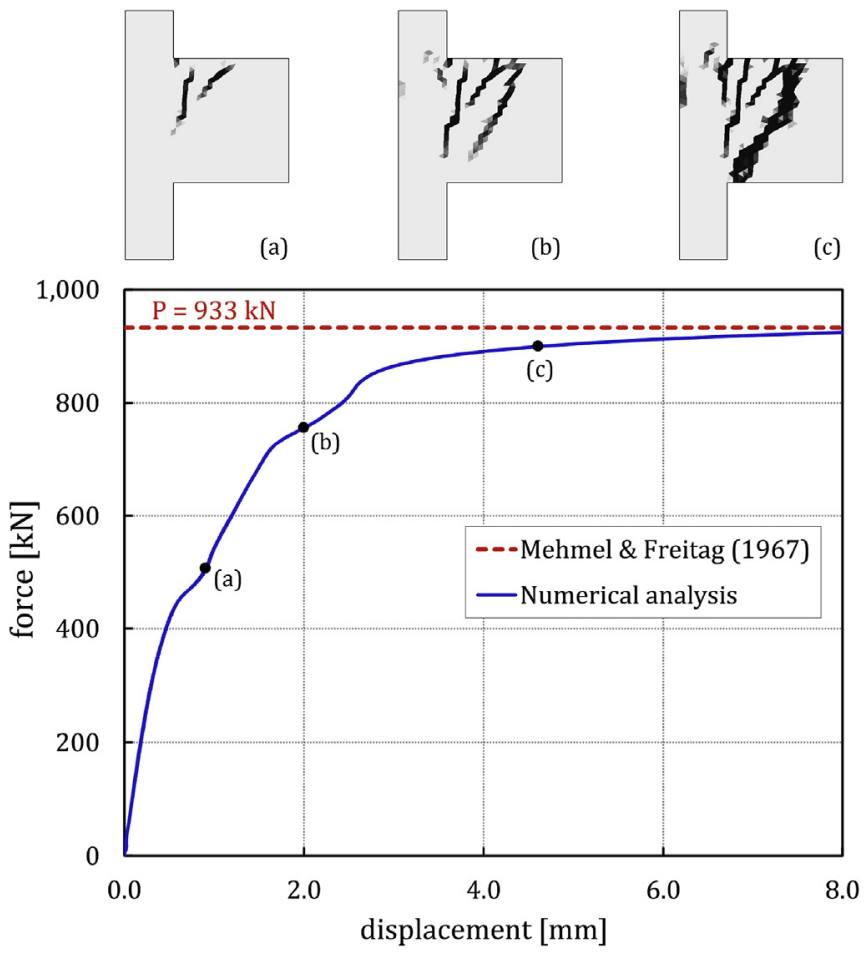

Fig. 24. Force versus displacement: experimental and numerical results.

forced concrete panels, respectively. In both cases, the experimental and numerical results are in good agreement.

Cracking initiates at the inner corner and then propagates towards the opposite side of the L-shaped specimen. The crack patterns obtained in both cases are illustrated in Fig. 22 for a vertical displacement of $1 \mathrm{~mm}$ and $10 \mathrm{~mm}$, respectively for the plain and reinforced concrete panels. As can be seen, the results are also in very good agreement with the experimental data showing the ability of the proposed approach to predict the process of crack localization and propagation.

\subsection{Reinforced concrete corbel}

This section presents 3D numerical analysis of a reinforced concrete corbel experimentally tested by Mehmel and Freitag [28]. Fig. 23(a) illustrates the test set-up and geometry, while the reinforcement layout is depicted in Fig. 23(b). Using symmetry conditions, only one-fourth of the corbel is analyzed, as represented in Fig. 23(c).

The finite element mesh employed in this example is the same shown earlier in Fig. 1, including the definition of CFEs.

The concrete is discretized using 53,665 four-noded tetrahedral finite elements. Their constitutive behavior is simulated using the continuum damage model presented in section 3.2. The rebars are discretized by 1681 two-noded truss finite elements with an elastic perfectly plastic model. The material parameters adopted for the constitutive models are summarized in Tables 4 and 5. The rigid coupling scheme is used to represent the perfect bond between the reinforcement and concrete, using 1701 five-noded tetrahedral coupling finite elements (see Fig. 1(c) and (d)) with elastic coupling parameters of $\widetilde{C}=10^{9} \mathrm{~N} / \mathrm{mm}$.

Fig. 24 shows the applied force versus displacement. As can be seen, the numerical model provides a good prediction of the experimental ultimate load capacity. The crack propagation process is also depicted for three different level of loads (Fig. 24(a), (b) and (c)). The comparison with the experimental pattern at the ultimate load of $P=933 \mathrm{kN}$ shows the good match obtained - see Fig. 25. The distribution of the normal stress in the steel reinforcements is also shown in this figure.

\section{Concluding remarks}

The present paper presents an alternative numerical formulation for modeling the mechanical behavior of reinforced concrete structures. The main novelty lies in the discrete representation of the rebars based on coupling finite elements.

The formulation is generalized for the cases of perfect bond - rigid coupling scheme - and loss of bond - non-rigid coupling scheme. A damage model is proposed to describe the bond-slip behavior using the recommendations of the CEB fib Model Code [25]. The concrete behavior is simulated adopting a continuum damage model with distinct damage variables for both tension and compression. Such model can be easily calibrated based on basic parameters obtained from experimental characterisation tests.

Each component of the proposed numerical approach was presented and validated progressively using three small case studies. First, a quarter cylinder with curved reinforcing layers was simulated to study the versatility when dealing with curved geometries and to assess the performance of the rigid coupling scheme. The results obtained were in good agreement with those by Elwi and Hrudey [17] obtained using a different numerical technique to embed the rebars. Next, a pullout test allowed to address the interaction between concrete and rebars using

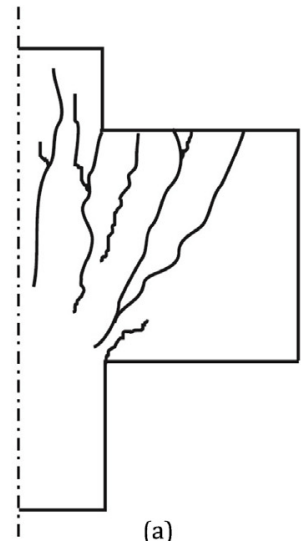

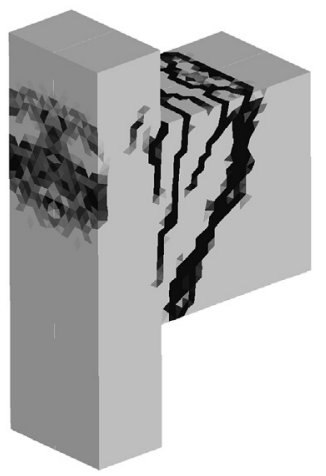

(b)

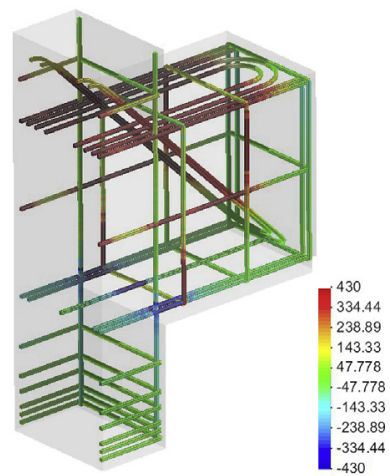

(c)

Fig. 25. Numerical versus experimental results for $P=933 \mathrm{kN}$ : (a) experimental crack pattern; (b) numerical crack pattern; and (c) normal stress in steel reinforcements (MPa). 
the bond-slip relation defined by the CEB fib Model Code [25]. Finally, an uniaxial tension and compression test was performed to assess the main features of the concrete damage model.

Three complex numerical applications were then selected to assess the performance of the proposed model in real concrete structures. First, a tension stiffening test allowed to assess the mesh sensitivity of the bond-slip model. Results obtained showed that the numerical model is able to adequately capture the bond-slip response and its interaction with the crack patterns. It was also shown that the results obtained are independent of the mesh size.

In the second application both plain and reinforced L-shaped panels were analyzed in a plane stress condition. Perfect bond was considered to connect the mesh of reinforcements to the concrete. In both specimens, the results obtained matched closely the experimental data by Winkler et al. [27].

The final 3D numerical analysis focused on a reinforced concrete corbel. The example had a complex arrangement of rebars with distinct diameters and inclinations. As was noted, the numerical model was able to predict the ultimate load capacity as well as the crack pattern obtained experimentally by Mehmel and Freitag [28].

It is also important to mention the limitations of the presented approach. The analyses are restricted to quasi-static loading and the failure process in concrete is modeled using a standard local continumm damage model. The rebars are represented by one-dimensional finite elements (truss elements) and, as consequence, bending and shear effects are not considered. In future works the authors intend to enhance the approach by considering beam elements, dynamic analysis, nonlocal damage models, as well as the discrete modeling of cracks.

Based on the results obtained from the numerical examples, it can be highlighted that the model presented to embed reinforcements based on CFEs effectively allows handling complex meshes for concrete and reinforcements independently. Although the meshes can be nonconforming, their interaction is adequately represented. The proposed formulation does not increase the degrees of freedom of the global problem and effectively avoids the need for special algorithms to detect the length of the reinforcement embedded in each parent element, while simultaneously allowing for multiple reinforcement segments, with different directions, to be automatically included in each crossed element.

\section{Acknowledgments}

Luís A.G. Bitencourt Jr. wishes to acknowledge the financial support of the National Council for Scientific and Technological Development - CNPq (429552/2016-5), São Paulo Research Foundation - FAPESP (2009/07451-2 and 2012/05430-0) and Coordination for the Improvement of Higher Education Personnel - CAPES. Dias-da-Costa would also like to extend his acknowledgments to the Australian Research Council through its Discovery Early Career Researcher Award (DE150101703) and ARC Projects (DP140100529, LP140100591 and DP170104192), and from the Faculty of Engineering \& Information Technologies, from The University of Sydney, under the Faculty Research Cluster Program.

\section{References}

[1] D. Ngo, A.C. Scordelis, Finite element analysis of reinforced concrete beams, ACI Journal 64 (3) (1967) 152-163.

[2] A.H. Nilson, Nonlinear analysis of reinforced concrete by the finite element method, ACI Journal 65 (9) (1968) 757-766.

[3] B. Richard, F. Ragueneau, L. Adelaide, C. Cremona, A multi-fiber approach for modeling corroded reinforced concrete structures, Eur. J. Mech. Solid. 30 (6) (2011) 950-961, https://doi.org/10.1016/j.euromechsol.2011.06.002, http:// www.sciencedirect.com/science/article/pii/S0997753811000763.

[4] B. Richard, F. Ragueneau, C. Cremona, L. Adelaide, J.L. Tailhan, A three-dimensional steel/concrete interface model including corrosion effects, Eng.
Fract. Mech. 77 (6) (2010) 951-973, https://doi.org/10.1016/j.engfracmech.2010. 01.017, http://www.sciencedirect.com/science/article/pii/S0013794410000664.

[5] E.A. Rodrigues, O.L. Manzoli, L.A.G. Bitencourt Jr., P.G.C. Prazeres, T.N. Bittencourt, Failure behavior modeling of slender reinforced concrete columns subjected to eccentric load, Lat. Am. J. Solid. Struct. 12 (3) (2015) 520-541, https://doi.org/10.1590/1679-78251224.

[6] F. Barzegar, Analysis of RC membrane elements with anisotropic reinforcement, J. Struct. Eng. 115 (3) (1989) 647-665, https://doi.org/10.1061/(ASCE)07339445(1989)115:3(647).

[7] F. Vecchio, Reinforced concrete membrane element formulations, J. Struct. Eng. 116 (3) (1990) 730-750, https://doi.org/10.1061/(ASCE)0733-9445(1990)116: 3(730).

[8] M. Polak, F. Vecchio, Nonlinear analysis of reinforced-concrete shells, J. Struct. Eng. 119 (12) (1993) 3439-3462, https://doi.org/10.1061/(ASCE)07339445(1993)119:12(3439).

[9] D. Owen, J. Figueiras, F. Damjanic, Finite element analysis of reinforced and prestressed concrete structures including thermal loading, Comput. Meth. Appl. Mech. Eng. 41 (3) (1983) 323-366, https://doi.org/10.1016/00457825(83)90012-9.

[10] H.-T. Hu, W. Schnobrich, Nonlinear finite element analysis of reinforced concrete plates and shells under monotonic loading, Comput. Struct. 38 (5) (1991) 637-651, https://doi.org/10.1016/0045-7949(91)90015-E.

[11] A. Ramaswamy, F. Barzegar, G. Voyiadjis, Study of layering procedures in finite-element analysis of RC flexural and torsional elements, J. Struct. Eng. 121 (12) (1995), https://doi.org/10.1061/(ASCE)0733-9445(1995)121:12(1773).

[12] N. E-Mezaini, E. Çitipitioğlu, Finite element analysis of prestressed and reinforced concrete structures, J. Struct. Eng. 117 (10) (1991) 2851-2864, https://doi.org/ 10.1061/(ASCE)0733-9445(1991)117:10(2851).

[13] H. Schäfer, A contribution to the solution of contact problems with the aid of bond elements, Comput. Meth. Appl. Mech. Eng. 6 (3) (1975) 335-353, https://doi.org/ 10.1016/0045-7825(75)90025-0.

[14] D.V. Phillips, O.C. Zienkiewicz, Finite element non-linear analysis of concrete structures, in: Proceedings of the Institution of Civil Engineers, vol. 61, 1976, pp. 59-88.

[15] A.E. Elwi, D.W. Murray, Nonlinear Analysis of Axisymmetric Reinforced Concrete Structures, , Tech. Rep. 87, University of Alberta, Department of Civil \& Environmental Engineering, 1980.

[16] T. Chang, H. Taniguchi, W. Chen, Nonlinear finite element analysis of reinforced concrete panels, J. Struct. Eng. 113 (1) (1987) 122-140, https://doi.org/10.1061/ (ASCE)0733-9445(1987)113:1(122).

[17] A.E. Elwi, T.M. Hrudey, Finite element model for curved embedded reinforcement, J. Eng. Mech. 115 (4) (1989) 740-754, https://doi.org/10.1061/(ASCE)07339399(1989)115:4(740).

[18] Z.A. Al-Bayati, J.J. Fahed, Development of steel representation as embedded bars in concrete using finite element method, J. Eng. Dev. 12 (2) (2008) 111-126.

[19] Y.M. Cheng, Y. Fan, Modeling of reinforcement in concrete and reinforcement confinement coefficient, Finite Elem. Anal. Des. 13 (4) (1993) 271-284, https:// doi.org/10.1016/0168-874X(93)90044-Q, http://www.sciencedirect.com/ science/article/pii/0168874X9390044Q.

[20] F. Barzegar, S. Maddipudi, Generating reinforcement in fe modeling of concrete structures, J. Struct. Eng. 120 (5) (1994) 1656-1662.

[21] F. Barzegar, S. Maddipudi, Three-dimensional modeling of concrete structures. ii: reinforced concrete, J. Struct. Eng. 123 (10) (1997) 1347-1356, https://doi.org/ 10.1061/(ASCE)0733-9445(1997)123:10(1347), http://link.aip.org/link/?QST/ $123 / 1347 / 1$

[22] G. Markou, M. Papadrakakis, An efficient generation method of embedded reinforcement in hexahedral elements for reinforced concrete simulations, Adv. Eng. Soft. 45 (1) (2012) 175-187, https://doi.org/10.1016/j.advengsoft.2011.09. 025, http://www.sciencedirect.com/science/article/pii/S096599781100264X.

[23] O.L. Manzoli, J. Oliver, A. Huespe, G. Diaz, A mixture theory based method for three-dimensional modeling of reinforced concrete members with embedded crack finite elements, Comput. Concr. 5 (6) (2008) 401-416.

[24] L.A.G. Bitencourt Jr., O.L. Manzoli, P.G.C. Prazeres, E.A. Rodrigues, T.N. Bittencourt, A coupling technique for non-matching finite element meshes, Comput. Meth. Appl. Mech. Eng. 290 (2015) 19-44, https://doi.org/10.1016/j cma.2015.02.025, http://www.sciencedirect.com/science/article/pii/ S0045782515000870.

[25] fib Model Code For concrete structures 2010, International Federation for Concrete Structures, Ernst \& Soh, Lausanne, 2013.

[26] M. Cervera, J. Oliver, O. Manzoli, A rate-dependent isotropic damage model for the seismic analysis of concrete dams, Earthq. Eng. Struct. Dynam. 25 (9) (1996) 987-1010, doi:10.1002/(SICI)1096-9845(199609)25:9<987::AID-EQE599> 3.0.CO;2-X.

[27] B. Winkler, G. Hofstetter, G. Niederwanger, Experimental verification of a constitutive model for concrete cracking, Proc. IME J. Mater. Des. Appl. 215 (2) (2001) 75-86.

[28] A. Mehmel, W. Freitag, Tragfahigkeitsversuche an stahlbetonkonsolen, Bauingenieur 42 (1967) 362-369. 